\title{
REVIEW OF LAMINAR CONVECTION HEAT-TRANSFER WITH MICROENCAPSULATED PHASE CHANGE MATERIAL SLURRY
}

\author{
Shiwei Chen ${ }^{1, \mathrm{a}}$, Zhongzhu Qiu ${ }^{1, \mathrm{~b}}$, Fengjiao Liu $^{1, \mathrm{c}}$ and Jin Xing ${ }^{2, \mathrm{~d}}$ \\ ${ }^{1}$ Shanghai University of Electric Power, Shanghai 200090, China \\ ${ }^{2}$ Binzhou University, Shandong 256600, China \\ acsw0923@126.com, b1050079260@qq.com
}

Keywords: MPCMS, laminar heat transfer, heat transfer correlations

\begin{abstract}
This paper mainly makes introduction about microencapsulated phase change material slurry (MPCMS).Microencapsulated phase change material slurry (MPCS ) can serve as both the heat transfer fluids and energy storage media, consequently, they are potentially applicable to the thermal systems. The preparations, thermal properties and several correlations of heat transfer in laminar flow of MEPCMS are also listed. In addition, it is found that the main influence factors for heat transfer effective is the bulk Stefan number.
\end{abstract}

\section{Introduction}

In the last 40 years, lots of efforts have been made to enhance the energy storage capacity of structural material for solar and building applications by integrating the PCM directly into the other materials, e.g. wallboard, concrete blocks, under floor heating system, etc.[1] However, the thermal performances of such applications are not significant due to the low thermal conductivity of PCM [2].

Several methods for generating PCM particles have been investigated for various thermal-energy applications. In recent years, a new approach was proposed, in which the phase change material was microencapsulated and suspended in a single-phase heat transfer fluid to form microencapsulated phase change material (MPCM) slurry [3]. These suspensions benefit from a number of special features including (a) relatively low variations in operating temperatures of systems using such fluids due to energy absorption at approximately constant temperature, (b) lower pumping power requirements due to the increased heat capacity [4], (c) high heat transfer rates to the phase change material due to large surface area to volume ratio, (d) the enhanced thermal conductivity of slurry leading to increased heat transfer to the suspension [5], and (e) the reduction/elimination of incongruent melting and phase separation[6]This paper presents a review of the research progress on MPCS as the heat-transfer fluids in laminar convection heat transfer..The review has been arranged into some sections including: the features of MPCM, the main parameters for heat transfer and the equation.

\section{Microencapsulated phase change material slurry (MPCS)}

Microencapsulation is a kind of tiny particles (about 0.1-100 $\mu$ m diameter) in which the core material is enveloped by a thin layer of shell.

There are several methods which can be used to produce microencapsulated particles, such as spray-drying and coacervation. Figure 1 is a photomicrograph of the MCPCM particles made by different methods. It can be seen from the surface morphology that the shape of MPCM is uniformly spherical and the surface is very smooth without edges and dents, which implies that fluidity is good if the MPCM is dispersed into the carrier fluids, such as water.

When the MPCM is dispersed into the carrier fluid, e.g. water, a kind of suspension named as microencapsulated phase change slurry (MPCS) is formed. During the fabrication process, an appropriate amount of surfactants are normally used for helping MPCM well disperse into the carrier fluid and increase the lifetime of MPCS [7]. In comparison of conventional phase change 
material slurries (PCS), better heat transfer performance can be achieved due to the relatively large surface area to volume of MPCM. Therefore, MPCS can be used as both thermal energy storage and heat transfer media.
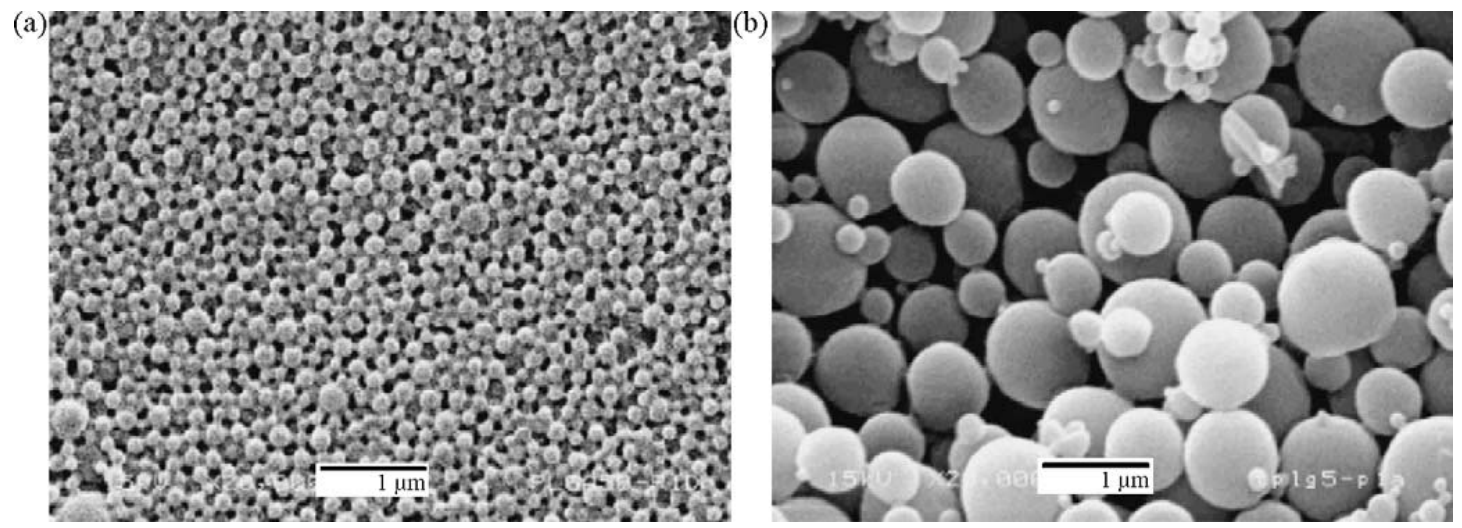

Fig. 1. Typical SEM pictures of MPCM made by different methods [3]. (a) By spray-drying and (b) by coacervation.

\section{Thermal and physical properties of MPCS}

The thermal and physical properties of MPCS are very different from those the MEPCM materials and carrier fluids. Due to the relatively large surface area to volume of MPCM, better heat transfer performance can be achieved. Thus, MPCS can serve as both the energy storage and heat transfer media, in addition, the agglomeration of MPCM can be avoided due to the encapsulation. It is obvious that, however, the heat transfer and fluid flow characteristics are very important for the MPCS system design. Due to the relatively large surface area to volume of MPCM, better heat transfer performance can be achieved.

The thermal conductivity of the microcapsule was calculated based on the composite sphere approach[8], as follows:

$$
\frac{1}{\mathrm{k}_{\mathrm{p}} \mathrm{d}_{\mathrm{p}}}=\frac{1}{\mathrm{k}_{\mathrm{c}} \mathrm{d}_{\mathrm{c}}}+\frac{\mathrm{d}_{\mathrm{p}}-\mathrm{d}_{\mathrm{c}}}{\mathrm{k}_{\mathrm{w}} \mathrm{d}_{\mathrm{p}} \mathrm{d}_{\mathrm{c}}}
$$

where $\mathrm{k}_{\mathrm{p}}$ is the thermal conductivity of the MPCM particle and $\mathrm{k}_{\mathrm{w}}$ the thermal conductivity of the water.

The thermal conductivity of the static MPCS with low volume fraction can be evaluated by Maxwell's equation formulate as

$$
k_{s}=k_{f} \times \frac{2 k_{f}+k_{p}+2 \emptyset\left(k_{p}-k_{f}\right)}{2 k_{f}+k_{p}-\emptyset\left(k_{p}-k_{f}\right)}
$$

where $\mathrm{k}_{\mathrm{s}}$ and $\mathrm{k}_{\mathrm{f}}$ are the thermal conductivities of the bulk slurry and carrier fluid, and $\mathrm{f}$ is the volume fraction of MPCM. The thermal conductivity of the MPCM particle $k_{p}$ can be estimated by a correlation describing composite sphere feature [9].

Due to the interaction between the particle and fluid the effective thermal conductivity is generally higher than that predicted by Maxwell's equation. Then, a general correlation summarized can be applied depending on the particle Peclet number

$$
\frac{k_{e}}{k_{s}}=1+B \emptyset P e_{P}^{m}
$$

where $\mathrm{k}_{\mathrm{e}}$ is the effective thermal conductivity of the MPCS, $P e_{p}=\vec{e} \cdot d^{2} / \alpha_{f}$ is the particle Peclet number, $\vec{e}$ is the shear rate, $\alpha_{f}$ is the thermal diffusivity, and the values for $B$ and $m$ depend on the particle Peclet number

The viscosity of MPCS is important in determining the pressure drop. Yamagishi et al. [10] indicated that the MPCS showed the Newtonian fluid feature and the apparent viscosity was about 1.5-10 times of the water as the mass fraction of MPCM increased from about 5 to $30 \%$.

Vand et al.[11] uses the following equation to estimate the viscosity of the slurry.

$\frac{\mu_{s}}{\mu_{f}}=\left(1-\emptyset-\mathrm{A} \emptyset^{2}\right)^{-2.5}$

where $\mathrm{A}$ is a parameter depending on the shape and rigidity of the particles. 
The specific heat of the MPCS without phase change can be simply estimated from the mass and energy balance approach which can be formulated as

$$
C_{P, s}=w_{p c m} C_{p c m}+w_{\text {shell }} C_{\text {shell }}+w_{f} C_{f}
$$

where $\mathrm{w}_{\mathrm{pcm}}, \mathrm{w}_{\text {shell }}$ and $\mathrm{w}_{\mathrm{f}}$ are the mass fractions of the PCM, shell and carrier fluid, respectively, and $\mathrm{w}_{\mathrm{pcm}}+\mathrm{w}_{\text {shell }}+\mathrm{w}_{\mathrm{f}}=1$.

\section{Flow and heat transfer characteristics of MPCS}

Charunyakorn et al. [8] proposed a theoretical model to study the forced convection heat transfer of MPCS. The results showed that the bulk Stephan number and particle volume fraction were the most important parameters which affected the heat transfer of MPCS.

Goel et al. [12] conducted experiments to investigate the laminar forced convection heat transfer of MPCS with the Reynolds numbers were about 200 and 1000. The experimental results indicated that about $50 \%$ reduction in wall temperature could be realized by using MPCS compared with single phase fluid flow a the Stephan number, defined in the following equation, was the most dominant parameter in the convection heat transfer similar to the results of Charunyakorn et al. [8].

$$
\text { Ste }=\frac{C_{p, s}\left|q_{w} R / k_{s}\right|}{\emptyset_{p} L \rho_{p} / \rho_{s}}
$$

Alvarado [13] showed that MPCM (n-tetradecane as the core material) with the diameter of smaller than $10 \mu \mathrm{m}$ could be endurable and impact-resistant by using cavity pump to do the durability test.

Xichun Wang and Jianlei Niu [14] investigate the heat transfer characteristics of MEPCMS flow in a horizontal tube, they proposed a heat transfer correlation for the slurry under laminar condition.

$$
N u_{m}=0.8148 \operatorname{Re}_{m}^{0.4593} \mathrm{Pr}_{m}^{0.4836} S t e^{-0.1277}\left[\left(L_{1}+L_{2}\right) / D\right]^{0.3059}
$$

The present experimental data for $60<\operatorname{Re}_{\mathrm{m}}<2200,12<\operatorname{Pr}_{\mathrm{m}}<73,0.05<\mathrm{w}_{\mathrm{p}}<0.276$. where $\mathrm{w}_{\mathrm{p}}$ is the mass fraction of MPCM particles in the slurry, [(L1 + L 2) / D] is phase change region length .

\section{Conclusions}

Laminar convection heat-transfer with microencapsulated phase change material slurry varied significant along the flow direction of the slurry. It is found that the heat transfer coefficients were always higher than those of single-phase fluid because of the latent heat effect and microconvective thermal conductivity enhancement. A numerical study shows that the Stefan number is the main parameter that has a significant impact on the heat transfer for typical phase change material suspensions and operating conditions.

Several laminar heat transfer correlations were proposed by other researchers, which predicts the heat transfer data. Those conclusions show that Stefan number is the most parameter that has a significant impact on the laminar heat transfer for typical phase change material slurry.

\section{Acknowledgements}

The author appreciate the financial support from the Shanghai Engineering Research Center of Power Generation Environment Protection

\section{References}

[1] M.M. Farid, A.M. Khudhair, S.A.K. Razack, A.A. Hallaj, A review on phase change energy storage: materials and applications, Energy Convers. Manage. 45 (2004) 1597-1615.

[2] F. Wang, G. Maidment, J. Missenden, R. Tozer, A review of research concerning the use of PCMs in air conditioning and refrigeration engineering, Adv. Build. Technol. 2 (2002) 1273-1280. 
[3] K.E. Kasza, M.M. Chen, Improvement of the performance of solar energy or waste heat utilization systems by using phase-change slurry as an enhancement heat-transfer storage fluid, J. Solar Energy Eng.107 (1985) 229-236.

[4] K. E. Kasza and M. M. Chen, Development of enhanced heat transfer/transport/storage slurries for thermal system improvement, ANL-82-50, Argonne National Lab., Illinois (1982).

[5] H. G. Leal, On the effective conductivity of dilute suspensions of spherical drops in the limit of low Peclet number, Chem. Engng Commun. 1,21 31 (1973).

[6] R. Hart and F. Thornton, Microencapsulation of phase change materials, Final Report Contract No. 82-80. Ohio Department of Energy, Ohio (1982).

[7] C.Y.ZHAO and G.H. Zhang Review on microencapsulated phase change materials (MEPCMs): Fabrication, characterization and applications. Renewable and Sustainable Energy Reviews,15 (2011) 3813-3832

[8] Charunyakorn P, Sengupta S, Roy SK. Forced convection heat transfer in microencapsulated phase change material slurries: flow in circular ducts. Int J Heat Mass Transfer 1991;34(3):819-33.

[9] Guyer EC, Brownell DL. Handbook of applied thermal design. New York: McGraw-Hill; 1998

[10] Yamagishi Y, Takeuchi H, Pyatenko AT, Kayukawa N. Characteristics of microencapsulated PCM slurry as a heat-transfer fluid. AIChE Journal 1999;45(4): 696-707.

[11] Vand V. Theory of viscosity of concentrated suspensions. Nature 1945;155:364-5.

[12] Goel M, Roy SK, Sengupta S. Laminar forced convection heat transfer in microcapsulated phase change material suspensions. International Journal of Heat and Mass Transfer 1994;37(4):593-604.

[13] Alvarado JL, Marsh C, Sohn C, Phetteplace G, Newell T. Thermal performance of microencapsulated phase change material slurry in turbulent flow under constan heat flux. International Journal of Heat andMass Transfer 2007;50(9-10):1938-52.

[14] Xichun Wang ,Jianlei Niu, Yi Li. Heat Transfer of Microencapsulated PCM Slurry Flow in a Circular Tube.[J] AIChE Journal,2008,54(4)1110-1120. 